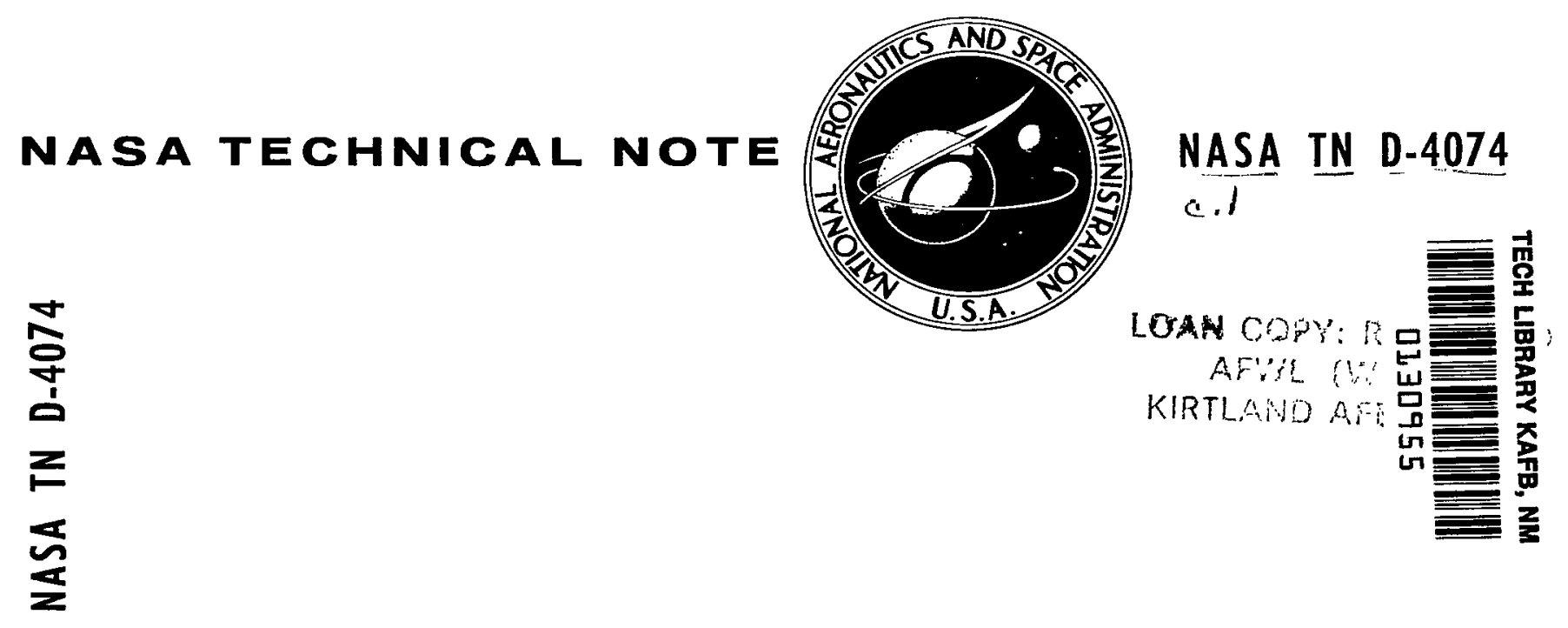

\title{
SIMULATION OF \\ DEPLOYMENT DYNAMICS \\ OF SPINNING SPACECRAFT
}

by William E. Lang and George H. Honeycutt Goddard Space Flight Center Greenbelt, $M d$. 
SIMULATION OF DEPLOYMENT DYNAMICS

OF SPINNING SPACECRAFT

By William E. Lang and George H. Honeycutt

Goddard Space Flight Center

Greenbelt, Md.

NATIONAL AERONAUTICS AND SPACE ADMINISTRATION

For sale by the Clearinghouse for Federal Scientific and Technical Informotion

Springfield, Virginia 22151 - CFSTI price $\$ 3.00$ 


\begin{abstract}
The evolution of spacecraft of optimum design and acceptable reliability requires knowledge of in-flight dynamic behavior during configuration changes. Systems having radially telescoping or folded appendages are analyzed. Neither system is completely amenable to closed form solution, but analog or digital computer techniques can be applied to specific cases. Digital techniques compare favorably with analog methods for this application. Coriolis effects are significant for rapid deployments. Behavior parameters can be related to system geometry in closed form but time histories require iteration. Telescoping systems are basically irreversible and folding systems cyclic but both are terminated in practice by latching devices. Solutions of the two basic systems may be rationally extrapolated to evaluate adequately more complex systems. Finally, pertinent test experience is summarized. When centrifugal appendage deployment is concurrent with despin, environmental simulation presents major problems including incorrect ambient gravity. Test methods are described with particular attention to gravity compensating techniques. Test results correlate acceptably with analytical predictions; however, it is concluded that analysis and testing should be complementary rather than mutually exclusive.
\end{abstract}




\section{CONTENTS}

Abstract......................... ii

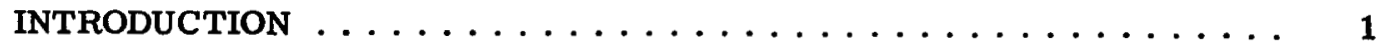

GENERAL PRINCIPLES ...................... 2

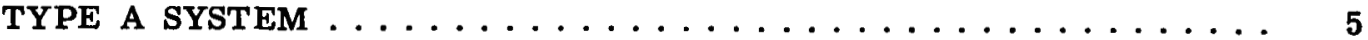

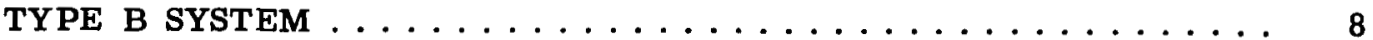

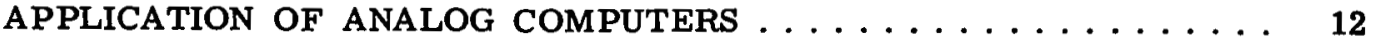

Type A System ........................... 12

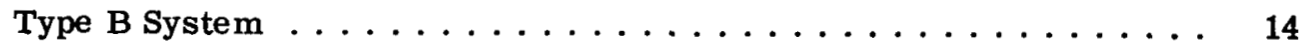

APPLICATION OF DIGITAL COMPUTERS . . . . . . . . . . . 14

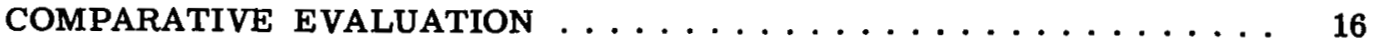

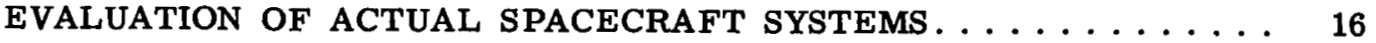

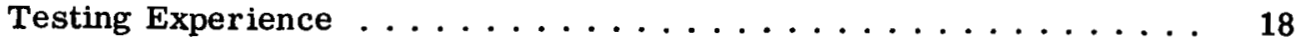

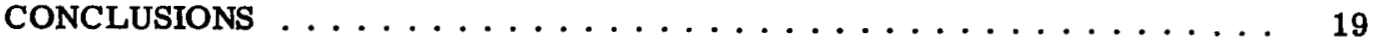

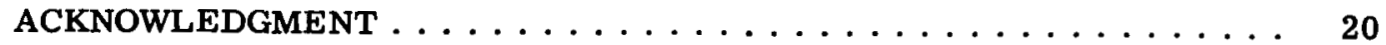

References ........................... 20

Appendix A-Influence of Central Body to Appendage Mass Ratio on Deployment Dynamics . . . . . . . . . . . . . . 21 


\title{
SIMULATION OF DEPLOYMENT DYNAMICS OF SPINNING SPACECRAFT
}

\author{
by \\ William E. Lang and George H. Honeycutt \\ Goddard Space Flight Center
}

\section{INTRODUCTION}

Spin stabilized spacecraft often use the rotational energy of a constrained launch configuration to accomplish change to an orbital configuration of increased inertia and reduced spin rate by sequential or concurrent centrifugal deployment of various appendages. This paper is concerned with evaluation of the dynamic effects of such deployments, particularly the nonlinear transient phenomena generated during periods of variable inertia. This evaluation requires mathematical simulation of system behavior and may also require laboratory tests of system performance with appropriate environmental control including the negation of gravity effects.

There has been prior recognition of appendage deployments as phenomena requiring both analytical and empirical study. Forsythe (References 1 and 2) and Gluck and Gale (References 3 and 4) have reported both theoretical analysis and testing of satellites with deployable appendages. Either analysis or testing may, for an actual spaceicraft design, involve numerous complicating factors which must be considered in the overall evaluation. Such factors might include complex mechanical linkages, distributed mass effects, initial precession of the system, devices to assist or retard deployment, detent features to control or delay deployment, frictional effects, and the need to consider possible failure mode performance. A more general factor, less tangible but of obvious practical significance, is that for any specific spacecraft design the primary objective is to establish confidence that it will perform acceptably, or that it will or may not, with deficiencies adequately identified to facilitate design improvements. Also, it is common to supplement even the most rigorous and complete analysis with some operational testing-primarily to verify quality control of components and assembly rather than to verify analysis. As either rigorous analyses or extensive test programs tend to produce much data which, however interesting, are irrelevant to the primary objective, along with a few values of critical significance, the purpose of this study is to develop methods to yield the critically significant values as quickly as possible.

The concomitant compromise of accuracy has to be considered but usually will be tolerable because design practice necessarily includes an operational safety factor - a marginal design would normally be considered unacceptable. To be more specific, failure modes for a satellite 
appendage deployment system would generally be either incomplete deployment or structural failure during deployment or at lockup into deployed position. The precise kinematics of deployment are often irrelevant to evaluation except insofar as they impose loads and stresses on the structure.

The following presentation will first discuss general principles involving formulations based on energy and momentum conservation, applicable to any case of centrifugal deployment of appendage from a spinning free body. Specific formulation defining transient behavior will then be developed for two basic systems. The first postulates symmetrical point masses receding radially from a spinning central body, and the second considers point masses constrained to circular arcs coplanar with the spin axis. These basic systems characterize spacecraft having radially telescoping and hinged appendages, respectively.

Various methods can be used to solve these basic systems, and the nature of the solutions will be discussed. Then the applicability of these solutions to the evaluation of more complex real systems will be considered. It is contended that the solutions of the basic systems may suffice by rational extrapolation to yield adequate evaluation of many more complex systems from the viewpoint of critical design criteria.

The evolution of testing facilities for subjecting spinning spacecraft to appendage deployment will be described and pertinent test experience will be reported. Particular attention will be given to the techniques which may be used to nullify gravity effects and to the significance of this factor. A discussion of testing facilities is included because theoretical analysis and test operations are considered as complementary rather than alternative evaluation techniques.

\section{GENERAL PRINCIPLES}

It is axiomatic that any spinning spacecraft whether of constant or varying inertia must maintain constant angular momentum. The total energy of the system must also be invariant and may or may not include energy contributed by springs or similar devices to assist deployment or expended in friction or deployment retarding devices. In many cases the energy involved is preponderantly kinetic, and assumption that only kinetic energy is involved greatly simplifies analysis.

Momentum and kinetic energy conservation yield two basic equations:

$$
\begin{gathered}
\mathrm{L}=\mathrm{I}_{0} \dot{\theta}_{0}=\mathrm{I}_{\mathrm{T}} \dot{\theta}_{\mathrm{T}} \quad \text { (Angular momentum) }, \\
2 \mathrm{~T}=\mathrm{I}_{0} \dot{\theta}_{0}^{2}=\mathrm{I}_{\mathrm{T}} \dot{\theta}_{\mathrm{T}}^{2}+\sum \mathrm{m}_{\mathrm{T}}^{2}+\sum \mathrm{m}_{\mathrm{T}}^{2} \text { (Kinet ic energy). }
\end{gathered}
$$

The subscripts designate initial and subsequent times; $I$ is the polar moment of inertia of the system, and $\dot{\theta}$ its angular velocity; " $m$ " designates masses of parts of the system: and $\dot{\mathrm{r}}$ and $\dot{\mathrm{x}}$ are velocity 
components of these masses, $r$ being radial from the spin axis and $x$ parallel to the spin axis. The third (tangential) component of absolute velocity is implicit in the $\mathrm{I}_{0} \dot{\theta}_{0}^{2}$ and $\mathrm{I}_{\mathrm{T}} \dot{\theta}_{\mathrm{T}}^{2}$ terms therefore, Equation 2 accounts for all the systems kinetic energy.

The specific formulation of the two basic equations will depend on the system geometry and will include variables $\ddot{\theta}, r$ and its first time derivative $\ddot{r}$. Here, $r$ will be the expanding radius of gyration of the deploying part of the system or possibly some angular parameter implicitly defining the mechanical restraints of the system geometry during deployment. It will include a constant (nondeploying) inertia plus $\mathrm{mr}^{2}$ terms for deploying masses. Velocities parallel to the spin axis (i.e., $\ddot{x}$ components) will be expressible without introducing any other variables by applying a third basic principle, namely that the linear momentum of the system in any direction and specifically along the spin axis, is constant and may be considered zero. (Any initial linear velocity of the entire system is irrelevant.) Therefore

$$
\sum_{m \dot{x}}=0
$$

It is also true that $\sum m \dot{r}=0$. In most cases this is intuitively obvious because of symmetry of the system about the spin axis (i.e., Appendages are usually designed as diametrically opposed similar pairs).

While a complete and general analytical solution of the problem thus formulated might be of academic interest, the questions of most practical significance are:

1. What will the spin rate be after deployment? This is obtained by substituting the deployed inertia value for $I_{T}$ in Equation 1.

2. How much energy will be dissipated by or in the structure due to deployment? The total amount is equal to $1 / 2\left(I_{0} \dot{\theta}_{0}^{2}-I_{F} \dot{\theta}_{F}^{2}\right)$, or $1 / 2 I_{0} \dot{\theta}_{0}{ }^{2}\left(1-I_{0} / I_{F}\right)$ where $I_{F}$ and $\dot{\theta}_{F}$ are the final inertia and spin rate. The loads resulting from transfer of this energy into friction loss and/ or strain energy depend on the geometry and compliance of the system. The total energy is proportional to the square of the initial spin rate.

3. The deployment involves transfer of momentum from the central body, which slows down, into the appendages, which speed up. The resulting tangential accelerations cause a rectrograde "bending back" of the appendages. As this effect is not constant during the deployment, pertinent questions are:

(a) For what geometric configuration is the effect maximum?

(b) When the effect is maximum, what loads are imposed on the structure?

If the appendage mass " $m$ " can be considered concentrated at a point at variable radius $r$ from the spin axis, the basic Equations 1 and 2 may be written:

$$
\left(\mathrm{I}+\mathrm{mr}_{0}{ }^{2}\right) \dot{\theta}_{0}=\left(\mathrm{I}+\mathrm{m} \mathbf{r}^{2}\right) \dot{\theta}
$$




$$
\left(\mathrm{I}+\mathrm{mr} \mathrm{r}_{0}^{2}\right) \ddot{\theta}_{0}^{2}=\left(\mathrm{I}+\mathrm{mr} \mathrm{r}^{2}\right) \dot{\theta}^{2}+\mathrm{m}\left(\dot{\mathrm{r}}^{2}+\ddot{\mathrm{x}}^{2}\right)
$$

where $I$ is the invariant inertia of the central body, and $\ddot{x}$ will be expressible in terms of $r, \ddot{r}$ and constant parameters of the system.

From basic analysis of the kinematics of a particle with curvilinear motion, the tangential acceleration $a_{t}$ of the mass " $m$ " is given by

$$
\mathbf{a}_{\mathbf{t}}=\mathbf{r} \ddot{\theta}+2 \dot{\mathbf{r}} \dot{\theta}
$$

or

$$
a_{t}=\frac{-I \ddot{\theta}}{m r},
$$

where $2 \dot{\theta} \dot{\mathbf{r}}$ is the so-called Coriolis acceleration.

Equations 4 and 5 permit expressing $\dot{\theta}$ and $\dot{r}$ in terms of $r$ and the constants. Then $\mathrm{d} \dot{\theta} / \mathrm{d}_{\mathrm{T}}$ can be determined and also $\ddot{\theta}$ since $\ddot{\theta}=(\mathrm{d} \dot{\theta} / \mathrm{d} \mathrm{r}) \dot{\mathrm{r}}$. Therefore $\mathrm{a}_{\mathrm{t}}$ can be expressed as a function of $r$, and its maximum value and the value of $r$ for which it is maximum can be found by solving the equation $d_{t} / d r=0$ for $r$ and substituting the solution for $a_{t}$.

Since $m a_{t}$ is the tangential force acting on $m$ and can be determined for any value of $a_{t}$, the loads acting on the structure can be determined. The bending moment at the root section of the appendage will be $\mathrm{ma}_{\mathrm{t}} t$ where $\ell$ is the distance from the root of the appendage to the point where its mass is assumed concentrated. It should be noted that if $\ell$ is dependent on $r$, rather than constant, the maximum value of the bending moment will not coincide with the maximum value of $a_{t}$.

At this point the approach described has defined the angular and radial velocities and accelerations plus the tangential acceleration as functions of position (i.e., $\ddot{\theta}, \ddot{\theta}, \dot{r}, \vec{r}$, and $a_{t}$ as functions of $r$ ). The behavior of the system is completely defined except for the time factor.

4. The final question to be answered is "How rapidly does the deployment proceed and how long does it take?" Thus, it is necessary to express $r$ as a time function; once this is done the preceding derivations of $\ddot{\theta}, \dot{r}$, etc., can also be converted to time functions.

Since $\dot{r}=d r / d t$ and is a known function of $r$ then $d t / d r=1 / \dot{r}$. Separating the variables and defining integration over appropriate limits,

$$
t=\int_{t_{0}}^{t} d t=\int_{r_{0}}^{r} \frac{d r}{\ddot{r}},
$$


Therefore, the time to deploy to radius $r$ can be determined by evaluating the integral

$$
\int_{r_{0}}^{r}(\dot{r})^{-1} d r
$$

Unfortunately, even for the simple systems to be considered, this integral can be evaluated only by graphical integration, iterative, or computer techniques. The application of these general principles to two basic simple systems will now be considered.

\section{TYPE A SYSTEM}

This system is shown in Figure 1 and consists of a spinning spacecraft with moment of inertia $I$. It has two diametrically opposed point masses $\mathrm{m} / 2$ initially located at radius $r_{0}$. At time $t_{0}$ the masses are released and thereafter slide radially out from the spin axis along frictionless massless guides. This system resembles one with telescoping appendages.

The kinetic energy is given by

$$
\mathrm{T}=\frac{\mathrm{m}}{2}\left(\dot{\mathrm{r}}^{2}+\mathrm{r}^{2} \dot{\theta}^{2}\right)+\frac{1}{2} \mathrm{I} \dot{\theta}^{2},
$$

which is derived from Equation 2. I is the moment of inertia of the spacecraft without the masses. The equations of motion can be obtained by using Lagrange's equation (Reference 5) (with no potential energy):

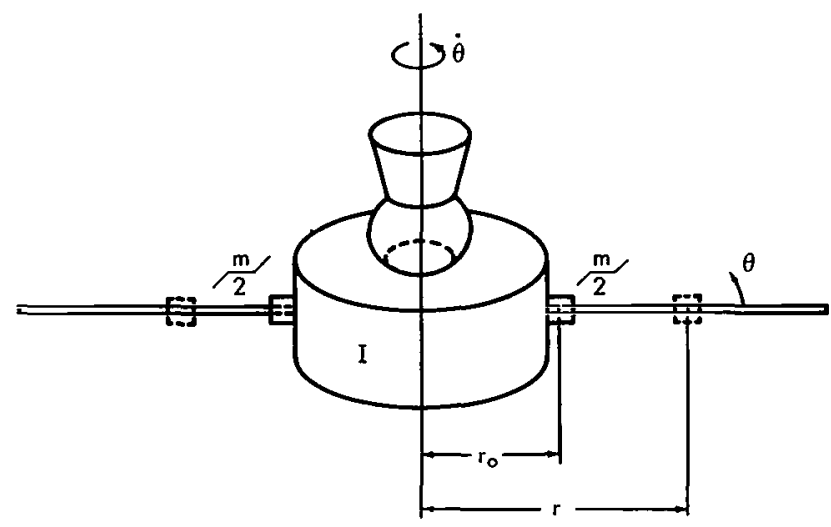

$$
\frac{d}{d t}\left(\frac{\partial T}{\partial \dot{q}_{k}}\right)-\frac{\partial T}{\partial q_{k}}=0
$$

Figure 1-Radially telescoping deployment system (type A).

where $q_{k}$ is each coordinate of the system in turn. Substituting Equation 9 into Equation 10 yields

$$
\begin{gathered}
\mathrm{m} \ddot{\mathbf{r}}-\mathrm{mr} \dot{\theta}^{2}=0, \\
\left(\mathrm{I}+\mathrm{mr}^{2}\right) \ddot{\theta}+2 \mathrm{mr} \dot{\mathrm{r}} \dot{\theta}=0 .
\end{gathered}
$$

The solution of Equations 11 and 12 for $r$ and $\ddot{\theta}$ will define the motion of the system. These equations are nonlinear and difficult to solve in closed form. However, by reverting to the momentum and energy equations much useful information can be obtained relatively easily without need for a 
complete solution. If a complete solution is desired, analog or digital computer methods must be used.

Methods for partial solutions will now be presented: from energy and momentum conservation,

$$
\begin{aligned}
& \left(\mathrm{I}+\mathrm{mr}_{0}{ }^{2}\right) \dot{\theta}_{0}=\left(\mathrm{I}+\mathrm{mr} \mathrm{r}^{2}\right) \dot{\theta}, \\
& \left(\mathrm{I}+\mathrm{mr}_{0}{ }^{2}\right) \ddot{\theta}_{0}^{2}=\left(\mathrm{I}+\mathrm{mr}^{2}\right) \dot{\theta}^{2}+\mathrm{mr^{2 }} .
\end{aligned}
$$

(Note that there is no relative motion parallel to the spin axis so no $\ddot{x}$ term.)

From Equations 13 and 14 the following can be derived:
(a) $\ddot{\theta}=\ddot{\theta}_{0}\left(\frac{I+m r_{0}^{2}}{I+m r^{2}}\right)$,
(b) $\frac{\mathrm{d} \dot{\theta}}{\mathrm{dr}}=-2 m r \dot{\theta}_{0}\left(\mathrm{I}+m r_{0}^{2}\right)\left(\mathrm{I}+m r^{2}\right)^{-2}$,
(c) $\quad \ddot{r}=\ddot{\theta}_{0}\left(I+m r_{0}^{2}\right)^{1 / 2}\left(r^{2}-r_{0}^{2}\right)^{1 / 2}\left(I+m r^{2}\right)^{-1 / 2}$,
(d) $\dddot{\mathbf{r}}=\dot{\mathbf{r}}^{2}$,
(e) $\ddot{\theta}=-2 m r \dot{\theta}_{0}^{2}\left(\mathrm{I}+\mathrm{mr}_{0}^{2}\right)^{3 / 2}\left(\mathrm{r}^{2}-\mathrm{r}_{0}^{2}\right)^{1 / 2}\left(\mathrm{I}+\mathrm{mr}{ }^{2}\right)^{-5 / 2}$.

Using these values and Equation 6, the tangential acceleration $a_{t}$ can be expressed as:

$$
a_{t}=r \ddot{\theta}+2 \dot{r} \dot{\theta}=2 I \dot{\theta}_{0}^{2}\left(I+m r_{0}^{2}\right)^{3 / 2}\left(r^{2}-r_{0}^{2}\right)^{1 / 2}\left(I+m r^{2}\right)^{-5 / 2}
$$

Setting $\mathrm{da}_{\mathrm{t}} / \mathrm{dr}=0$ yields

$$
r=\left(\frac{I+5 m r_{0}^{2}}{4 m}\right)^{1 / 2}
$$

and by substitution the maximum $a_{t}$ is

$$
a_{\mathrm{t} \max }=0.572 \mathrm{I} \dot{\theta}_{0}^{2}\left(\mathrm{Im}+\mathrm{m}^{2} \mathrm{r}^{2}\right)^{-1 / 2} .
$$


From a load analysis viewpoint the maximum retrograde torque developed on the central body (i.e., mrat ) or the maximum bending moment (denoted as $B$ ) at the inboard end of the guide (i.e., $m_{t}\left[r-r_{0}\right]$ ) would be more significant. Since general solutions for $d\left(m r a_{t}\right) / d r=0$ or $d\left(\right.$ ma $_{t}\left(r-r_{0}\right) / d r=0$ cannot be conveniently formulated, these maxima could more easily be determined by graphically plotting $m r a_{t}$ and $m a_{t}\left(r-r_{0}\right)$ for appropriate ranges of values for $r$.

It is noteworthy that $a_{t}, m_{r} a_{t}$, and $m_{t}\left(r-r_{0}\right)$ values, which may be thought of as transient wrenching effects, are all basically proportional to the square of the initial spin rate.

With phenomena of interest defined as functions of $r$, it remains to express $r$ (and implicitly the other phenomena) as time functions.

The time $t$ for $m$ to deploy to radius $r$ is given by:

$$
t=\int_{r_{0}}^{r} \frac{d r}{\ddot{r}}=\left(r_{0} \dot{\theta}_{0}\right)^{-1}\left(\frac{I}{m r_{0}^{2}}+1\right)^{-1 / 2} \int_{r_{0}}^{r}\left[\frac{\frac{I}{m r_{0}^{2}}+\left(\frac{r}{r_{0}}\right)^{2}}{\left(\frac{r}{r_{0}}\right)^{2}-1}\right]^{1 / 2} d r
$$

It is noteworthy that for any particular system, deployment time is inversely proportional to initial spin rate. It is not possible to express the integral of Equation 17 in closed form, except for the singular cases where either I or $m$ are zero. However, an approximate evaluation can be made by graphical or iterative methods for any specific value of $r_{0}$ and the dimensionless parameter $\mathrm{I} / \mathrm{mr}_{0}{ }^{2}$. The computer solutions discussed later essentially performed this integration producing curves for $\mathbf{r}, \dot{\mathbf{r}}, \ddot{\mathbf{r}}, \ddot{\theta}, \ddot{\theta}$, etc.

A graphical integration approach encounters the difficulty that the integrand function

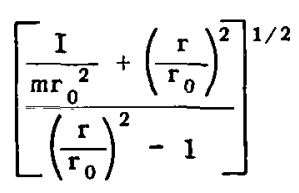

becomes infinite as $r$ approaches $r_{0}$. This problem can be circumvented by noting that for $r<r_{1}+\delta$ where $\delta \ll r_{0}, \dddot{r} \approx r_{0} \ddot{\theta}_{0}^{2}$. Therefore the time to deploy from $r_{0}$ to $r_{0}+\delta$ is essentially $\left(2 \delta / r_{0} \ddot{\theta}_{0}^{2}\right)^{1 / 2}$ and this time can be added as a constant to an integration from $r=\left[r_{0}+\delta\right]$ to the final value of $r$.

If either $I$ or $m$ are zero in Equation 17, closed form integration is possible. For $I=0$, or $I / \mathrm{mr}_{0}^{2}=0$, one may derive that $t=\ddot{\theta}_{0}^{-1}\left[\left(r / r_{0}\right)^{2}-1\right]^{1 / 2}$ or $r=r_{0}\left(1+\ddot{\theta}_{0}^{2} t^{2}\right)^{1 / 2}$ whereas for $\mathrm{m}=0$, or $\mathbf{I} / \mathbf{m r}_{\mathbf{0}}{ }^{2}=\infty$,

$$
\mathbf{t}=\dot{\theta}_{0}^{-1} \log _{e}\left(\frac{\mathrm{r}+\sqrt{\mathrm{r}^{2}-\mathrm{r}_{0}^{2}}}{\mathrm{r}_{0}}\right) \text {. }
$$


For real systems, numerical values of $I \gg m$ or $m \gg$ I would be unlikely; however, all real systems are between these extremes as demonstrated in Figures 9 and 10 and these closed form expressions were used to verify generalized analog computer solutions for $\mathrm{I} / \mathrm{mr}_{0}{ }^{2}=0$ and $\mathrm{I} / \mathrm{mr}_{0}{ }^{2}=1024$. (Computer operation precluded input of $\left.\mathrm{I} / \mathrm{mr}_{0}{ }^{2}=\infty\right)$. For $\mathrm{I} \gg \mathrm{m}$ or $\mathrm{m} \gg \mathrm{I}$ the relationship between $r$ and $t$ depends only on $\dot{\theta}_{0}$ and $r_{0}$.

The behavior of any Type A system is therefore predictable with relative ease even without the computer techniques which will be described later. At this point, it is interesting and instructive to examine the nature of solutions for a specific case which are presented in Figures 2 and 3 . For this example, $I=10.5$ slug- $\mathrm{ft}^{2}, \mathrm{~m}=0.25$ slugs, $r_{0}=1 \mathrm{ft} ., \ddot{\theta}_{0}=4.82 \mathrm{rad} / \mathrm{sec}$. Figure $2 \mathrm{presents}$

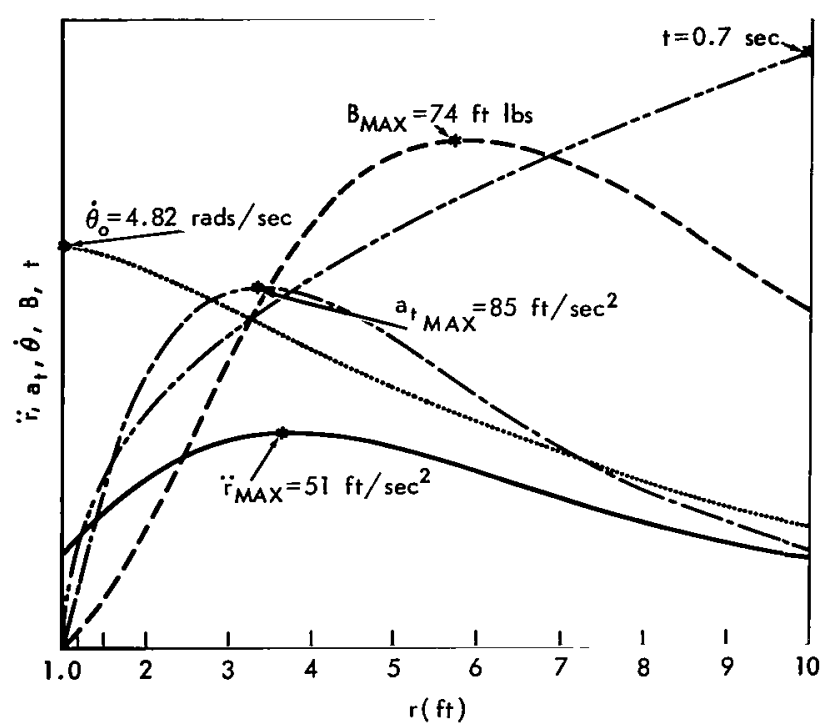

Figure 2-Type A system $\vec{r}, a_{t}, \dot{\theta}, B$ and time versus $r$.

$\ddot{r} ; a_{t}, \dot{\theta}, \mathrm{B}$ (the maximum bending moment at the inboard end of the guide, which is $\mathrm{ma}_{t}\left[\mathrm{r}-\mathrm{r}_{0}\right]$ ) and time, $t$, as functions of $r$. $r$ starts at $r_{0} \dot{\theta}_{0}^{2}$, increases to a maximum and then decreases. $a_{t}$ starts at zero, increases to a maximum defined by Equation 16, and then decreases. $\dot{\theta}$ decreases as the system inertia increases. $B$ rises from zero to a maximum and then decreases. Figure 3 presents the same parameters as time functions. The various maximum are nonsimultaneous and occur at different values of $r$.

\section{TYPE B SYSTEM}

This system is shown in Figure 4 and consists of a spinning spacecraft as before, but this time the masses are attached to massless booms of length $\ell$ which in turn are attached at radius $r_{0}$. At time $t_{0}$ the booms are released and thereafter swing out from the spin axis. The central body has mass $M$. The angle between the booms and the spin axis is denoted $a$ and is initially zero. Other symbols retain their significance in the prior discussion. 
The kinetic energy of this system is expressed by

$2 \mathrm{~T}=\mathrm{m}\left[\ell^{2} \dot{a}^{2}+\left(\mathrm{r}_{0}+\ell \sin a\right)^{2} \dot{\theta}^{2}+2 \dot{\alpha} \ell \sin a \ddot{\mathbf{x}}+\dot{\mathrm{x}}^{2}\right]$

$$
+M \dot{\mathbf{x}}^{2}+\mathbf{I} \ddot{\theta}^{2}
$$

where $\ddot{x}=-m \ell \ddot{a} \sin a / M+m$ and is the linear velocity of $M$.

If $\mathrm{M}$ is considered to be $\gg \mathrm{m}$, (see Appendix A) then the kinetic energy of this system is simplified:

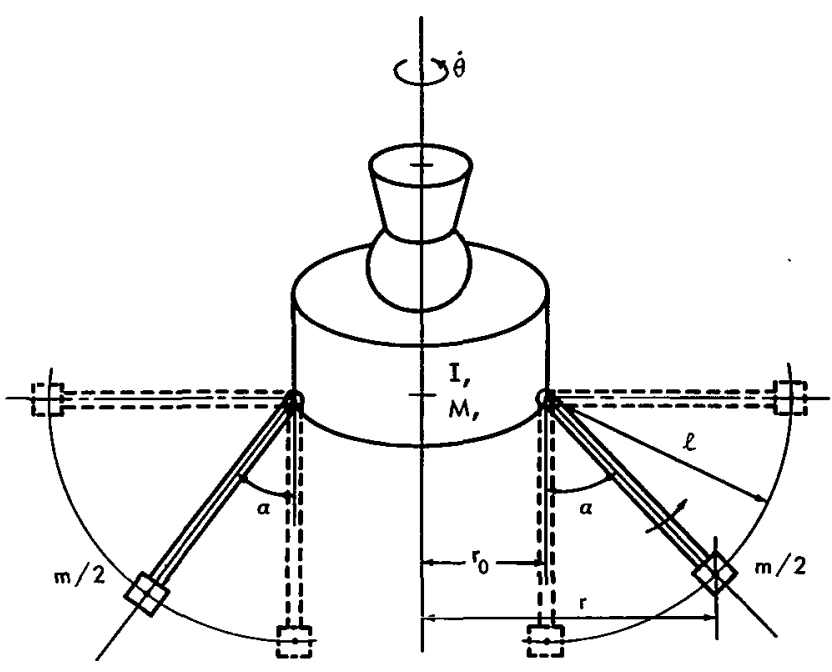

Figure 4-Hinged deployment system (Type B).

$$
2 \mathbf{T}=\mathbf{m}\left[\ell^{2} \ddot{\alpha}^{2}+\left(\mathrm{r}_{0}+\ell \sin \alpha\right)^{2} \dot{\theta}^{2}\right]+\mathbf{I} \dot{\theta}^{2} .
$$

Using Lagrange's Equation 10 with Equation 19:

$$
\begin{aligned}
& \left(\mathrm{I}+\mathrm{m} \mathrm{r}^{2}\right) \ddot{\theta}+2 m \ddot{\alpha} \ddot{\theta} \mathbf{r} \ell \cos \alpha=0 \text {, } \\
& \ell^{2} \ddot{a}-r \ell \cos \alpha \dot{\theta}^{2}=0 \text {, }
\end{aligned}
$$

where

$$
\mathbf{r}=\mathbf{r}_{0}+l \sin a .
$$

These equations are obviously more difficult than those for the Type A system but can still be solved with the help of an analog or digital computer. The analog computer used to solve the Type A system did not have enough multipliers for the Type B system equations so only the digital solution was obtained. As with the Type A system, it is possible to use basic principles to obtain partial solutions of considerable value.

This system differs from Type $A$ in two basic ways. Both $M$ and $m$ develop velocity components parallel to the spin axis; therefore, a rigorous formulation has to include the interchange of linear energy and momentum between them, consistent with the net energy and momentum of the whole system remaining constant. Also, while system $A$ is an open ended, irreversible phenomenon (the sliding weights would recede without theoretical limit with the spin rate decreasing asymptotically 
to zero), system $\mathrm{B}$ is basically periodic. The arms would flap repetitively through a $180^{\circ}$ arc, but it will suffice to limit the study to the first $90^{\circ}$ of travel.

Here a choice of geometric variable exists: either the radius $r$ to the mass $m$, or the angle $a$. Either one implicitly defines the other since $r=r_{0}+\ell \sin a$.

In terms of $r$, the basic equations can be derived so that

$$
\begin{gathered}
\left(\mathrm{I}+\mathrm{mr}_{0}{ }^{2}\right) \dot{\theta}_{0}=\left(\mathrm{I}+\mathrm{mr} \mathbf{r}^{2} \dot{\theta},\right. \\
\left(\mathrm{I}+\mathrm{mr}_{0}{ }^{2}\right) \dot{\theta}_{0}^{2}=\left(\mathrm{I}+\mathrm{mr}{ }^{2}\right) \dot{\theta}^{2}+\frac{\mathrm{m} \ell^{2} \dot{\mathrm{r}}^{2}}{\ell^{2}-\left(\mathrm{r}-\mathrm{r}_{0}\right)^{2}}\left[1-\frac{\mathrm{m}}{M+\mathrm{m}}\left(\frac{\mathrm{r}-\mathrm{r}_{0}}{\ell}\right)^{2}\right]
\end{gathered}
$$

These equations are based on Equations 4 and 5 with due consideration to the fact that $\sum_{\mathrm{m} \ddot{\mathrm{x}}=0}$ (Equation 3) and the geometric constraints of the system.

In terms of $a$, the equivalent equations are

$$
\begin{gathered}
\left(\mathrm{I}+\mathrm{mr}_{0}^{2}\right) \dot{\theta}_{0}=\left[\mathbf{I}+\mathrm{m}\left(\mathrm{r}_{0}+\ell \sin \alpha\right)^{2}\right] \dot{\theta}, \\
\left(\mathrm{I}+\mathrm{mr}_{0}^{2}\right) \dot{\theta}_{0}^{2}=\left[\mathrm{I}+\mathrm{m}\left(\mathrm{r}_{0}+\ell \sin \alpha\right)^{2}\right] \dot{\theta}^{2}+\mathrm{m} \ell^{2} \dot{\alpha}^{2}\left(1-\frac{\mathrm{m}}{\mathrm{M}+\mathrm{m}} \sin ^{2} a\right) .
\end{gathered}
$$

If $M \gg m$, as in many actual systems, the constant $m /(M+m)$ might reasonably be neglected, since it is essentially zero; but even then the formulation is harder to work with than that for the Type A system. The implications of the relative magnitudes of $M$ and $m$ are discussed in Appendix $A$.

The tangential acceleration $a_{t}$ can be expressed as

$$
a_{t}=\frac{2 I \dot{\theta}_{0}^{2}\left(I+m r_{0}^{2}\right)^{3 / 2}}{l\left(I+m r^{2}\right)^{5 / 2}}\left(r^{2}-r_{0}^{2}\right)^{1 / 2}\left[l^{2}-\left(r-r_{0}\right)^{2}\right]^{1 / 2}\left[1-\frac{m}{M+m}\left(\frac{r-r_{0}}{l}\right)^{2}\right]^{-1 / 2}
$$

The expression for $a_{t}$ in terms of $a$ is more cumbersome. The maximum value of $a_{t}$ may be found by trial substitution of values for $r$, since $d a_{t} / d r=0$ is not amenable to solution. For constants representative of many real systems, $a_{\operatorname{tmax}}$ occurs between $\alpha=20^{\circ}$ and $a=30^{\circ}$. The retrograde torque on the central body is $\mathrm{mra}_{t}$ and its maximum will not coincide with $a_{\operatorname{tmax}}$. However, the maximum bending moment across the hinge will coincide with $a_{t \max }$ and will equal $m \ell_{t_{\max }}$.

The wrenching effects are, as with Type A systems, basically proportional to the square of initial spin rate. 
The time $t$ for $m$ to deploy to radius $r=r_{t}$ (limited by conditions that $r_{t} \bar{z} r_{0}+\ell$ and $a_{t} \bar{\gtrless} 90^{\circ}$ is given by:

$$
t=\left(r_{0} \dot{\theta}_{0}\right)^{-1}\left(1+\frac{I}{m r_{0}^{2}}\right)^{-1 / 2} \int_{r_{0}}^{r_{t}}\left\{\frac{\left(\frac{I}{m r_{0}^{2}}+\frac{r^{2}}{r_{0}^{2}}\right)\left[l^{2}-\frac{m}{M+m}\left(r-r_{0}\right)^{2}\right]}{\left(\frac{r^{2}}{r_{0}^{2}}-1\right)\left[l^{2}-\left(r-r_{0}\right)^{2}\right]}\right\}^{1 / 2} d r
$$

It is noteworthy that, as for the Type A system, deployment time is inversely proportional to initial spin rate. Integration between the limits $r_{0}$ and $r_{t}=r_{0}+\ell$ would give the time to deploy through $90^{\circ}$.

In terms of $a$, the time to deploy to angle $a_{t} \leqq 90^{\circ}$ is

$$
t=\ell\left(r_{0} \dot{\theta}_{0}\right)^{-1}\left(1+\frac{I}{m r_{0}^{2}}\right)^{-1 / 2} \int_{0}^{\alpha}\left\{\frac{\left[\left(1+\frac{l}{r_{0}} \sin \alpha\right)^{2}+\frac{I}{m r_{0}^{2}}\right]\left(1-\frac{m}{M+m} \sin ^{2} a\right)}{\left(1+\frac{l}{r_{0}} \sin \alpha\right)^{2}-1}\right\} d \alpha
$$

Integration between limits of zero and $a_{t}=\pi / 2$ would give the time to deploy through $90^{\circ}$.

These time integrals cannot be evaluated in closed form. It is necessary to use graphical or iterative methods to obtain estimates for specific system parameters. In contrast to the Type A system, which can be characterized by a single dimensionless parameter $\left(I / \mathrm{mr}_{0}{ }^{2}\right)$ the Type $B$ system requires three such parameters, namely $I / \mathrm{mr}_{0}{ }^{2}, \ell / \mathrm{r}_{0}$, and $\mathrm{m} / \mathrm{M}+\mathrm{m}$ and they are mutually independent. Therefore, though a computer could be programmed to solve any particular system, a generalized solution, like that obtained for the Type A system by plotting families of curves for a wide range of $I / \mathrm{mr}_{0}^{2}$ values, is not feasible.

Figure 5 compares the time history of $r$ for a Type A system having the constants used in the previous example with the time histories of $r$ and $a$ for an equivalent Type B system having the same constants plus values of 4 feet for $\ell$ and $\infty$ for $M$. The $B$ system solutions were obtained by graphical and iterative methods and later confirmed by a digital computer per Figures 12, 13, and 14 (described in a later

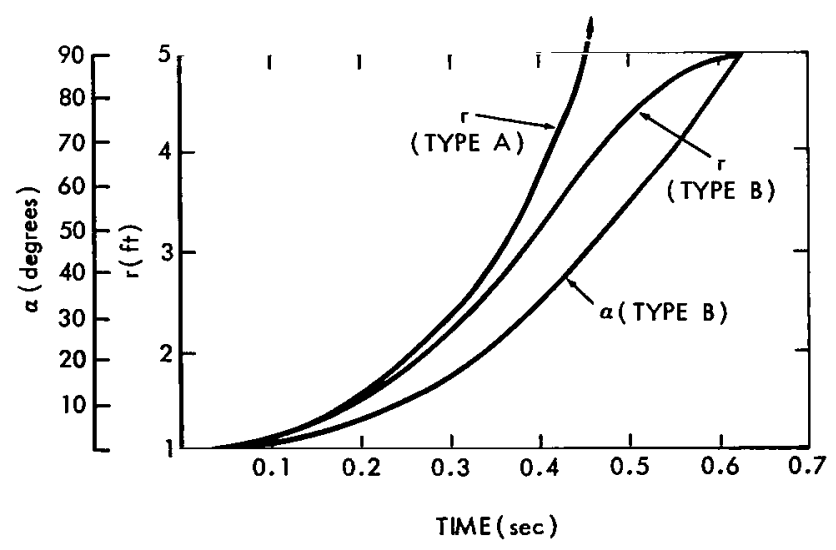

Figure 5- $r$ versus time compared for Type A and Type B systems. 
section). The B system deployment is slower but minimally so for the period immediately after release when $a$ is small.

If $M$ is assumed zero, the equations revert to the equivalent equations for the $A$ system; and a $B$ system with $M=0$ would in fact behave as an A system with the Type A history of $r$. A B system solution was also obtained and plotted for $M=10 \mathrm{~m}=2.5$ slugs. The resulting $\mathrm{r}$ versus time curve (not shown) deviated only slightly from the B system curve shown and moved closer to the A system curve with a final deployment time of 0.6135 seconds rather than 0.6232 seconds for $M=\infty$. The magnitude of $M$ also affects the tangential acceleration $T$ (per Equation 26). $T$ is less for infinite $M$ than for finite $M$, but the difference is very minor for $M \geqq 10 \mathrm{~m}$. The relative mass factor is discussed further in Appendix A.

\section{APPLICATION OF ANALOG COMPUTERS}

\section{Type A System}

The solution of Equations 11 and 12 can readily be obtained (Reference 6 ) using any general purpose analog computer providing the computer has enough amplifiers, integrators, multipliers, etc. (The analog computer used for all of the problems discussed here was the Electronic Associates Inc. PACE TR-48.)

Since Equations 11 and 12 reasonably describe the motion of a number of appendage deployment systems, it was decided to nondimensionalize the equations and get a general solution in the form of families of curves which could be entered directly for approximate solutions. This was accomplished by introducing the following non-dimensional variables ${ }^{\dagger}$ :

$$
\begin{aligned}
& \tau=\ddot{\theta}_{0} \mathrm{t} \quad \text { (non-dimensional time) } \\
& \mathbf{R}=\frac{\mathrm{r}}{\mathrm{r}_{0}} \quad \text { (non-dimensional radius) } \\
& \dot{\mathbf{R}}=\frac{\mathrm{d} \mathbf{R}}{\mathrm{d} \tau}=\frac{\ddot{\mathbf{r}}}{\mathbf{r}_{0} \dot{\theta}_{0}}, \quad \quad \quad \quad=\frac{\mathrm{d} \Theta}{\mathrm{d} \tau}=\frac{\dot{\theta}}{\dot{\theta}_{0}}, \\
& \ddot{\mathbf{R}}=\frac{\mathrm{d}^{2} \mathbf{R}}{\mathrm{d} \tau^{2}}=\frac{\dddot{\mathbf{r}}}{\mathbf{r}_{0} \dot{\theta}^{2}}, \quad \ddot{\Theta}=\frac{\mathrm{d}^{2} \Theta}{\mathrm{d} \tau^{2}}=\frac{\ddot{\theta}}{\dot{\theta}_{0}^{2}}, \\
& (\theta=\Theta) .
\end{aligned}
$$

\footnotetext{
$\dagger \theta$ is inherently non-dimensional, but it is desirable to use different symbols for all variables in the generalized problem with the

- superscripts denoting derivatives with respect to non-dimensional time $\tau$.
} 
Substituting these relationships into Equations 11 and 12 yields

$$
\begin{aligned}
& \mathbf{R}=\mathbf{R}^{2} \\
& \left(\frac{I}{m r_{0}^{2}}+R^{2}\right) \ddot{\Theta}+2 R \dot{R} \ddot{\theta}=0
\end{aligned}
$$

Equations 29 and 30 consist of dimensionless variables and one constant term $\mathrm{I} / \mathrm{mr}_{0}{ }^{2}$. This constant term defines the physical parameters of the spacecraft system, i.e., $I, m$, and $r_{0}$. Thus by solving equations 29 and 30 for a wide range of $1 / \mathrm{mr}_{0}^{2}$ values one can obtain a family of curves for each

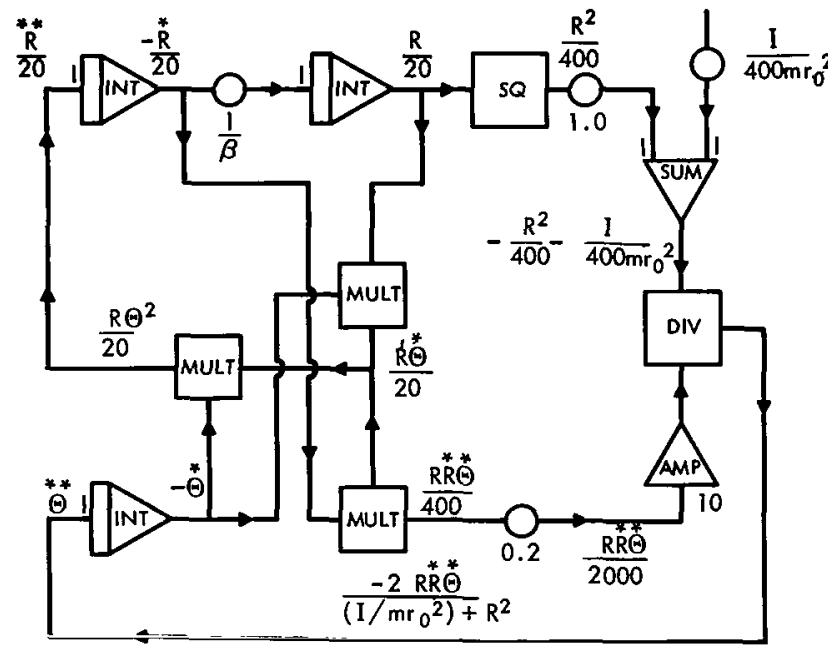

Figure 6-Analog computer schematic for generalized Type A system.

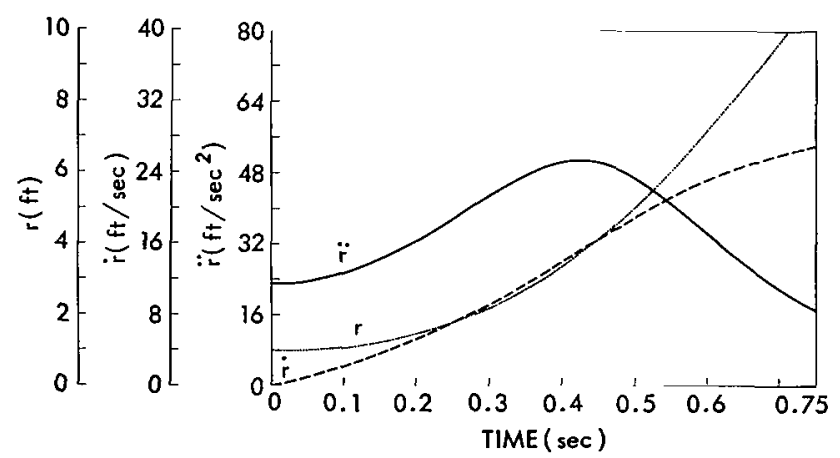

Figure 7-Specific analog computer solution for $r, \dot{r}$, and $\ddot{r}$ (Type A system). non-dimensional variable which can be transposed into the actual variables for any spacecraft that resembles the Type A system.

Figure 6 shows the programming schematic for the solution of Equations 29 and 30. As a check on the generalized solution a specific problem was also programmed using the same physical parameters used in the example of the preceding alternative method of solution; $I=10.5$ slug- $\mathrm{ft}^{2}, r_{0}=1$ foot, $\mathrm{m}=\mathbf{0 . 2 5}$ slugs, and $\dot{\theta}_{0}=4.82 \mathrm{rad} / \mathrm{sec}$., Figures 7 and 8 show the analog computer solution for this specific problem.

Figures 9 and 10 show the generalized analog computer solution for the families of $R$ and $\dot{\Theta}$ for values of $\mathrm{I} / \mathrm{mr}_{0}{ }^{2}$ from 0 to 1024 .

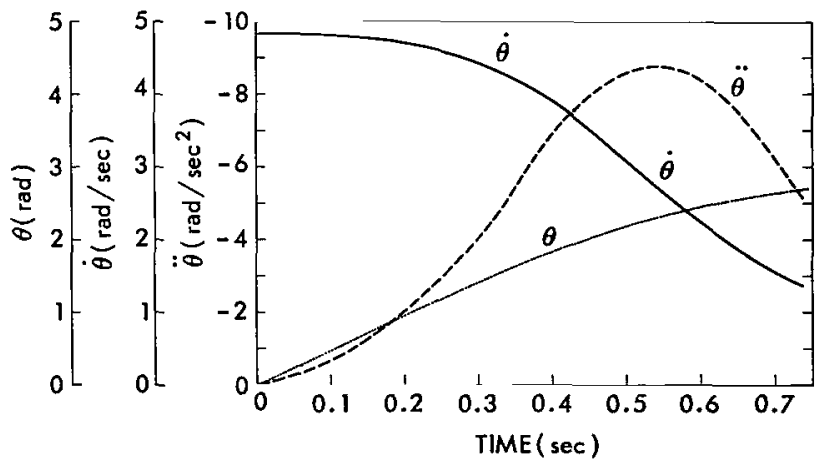

Figure 8-Specific analog computer solution for $\theta, \dot{\theta}$, and $\ddot{\theta}$ (Type A system). 


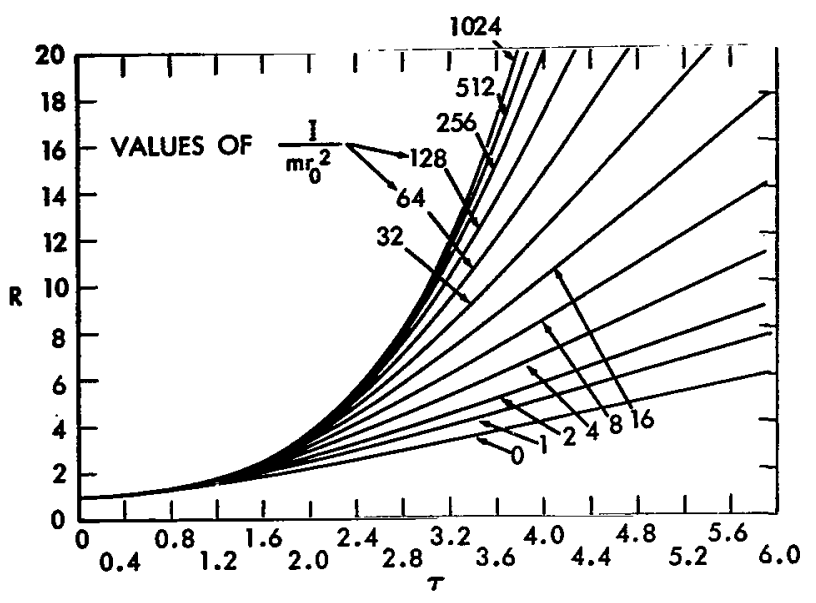

Figure 9-Generalized analog computer solution for $R$ (Type A system).

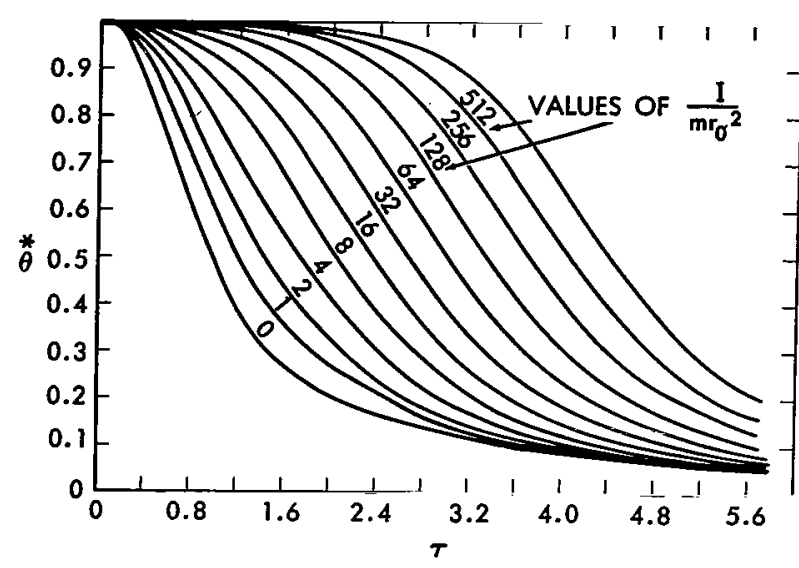

Figure 10-Generalized analog computer solution for $\dot{\mathbf{\theta}}$ (Type A system).

Using the generalized curves and working back to obtain the actual variables for the specific problem will give the same results, thus verifying the generalized solution. The results also correspond closely with those obtained for the same problem by the methods previously discussed, and shown in Figure 3.

\section{Type B System}

It would be interesting and informative to perform the same kind of analog computer solution for the Type B system. However, Equations 20 and 21 are more complicated than Equations 11 and 12 and any attempt to non-dimensionalize them results in the several independent constants, mentioned previously, which would made the number of family curves prohibitively large. In fact, even to solve a specific problem requires a number of sine-cosine generators and more multipliers than needed for the Type A system equations. The TR-48 computer did not have enough multipliers to solve this problem and since the MIMIC. Digital Simulation Program (Reference 7) was available no attempt was made to solve the equations with an analog computer. However, with a larger analog computer a specific spacecraft deployment problem could be readily handled.

\section{APPLICATION OF DIGITAL COMPUTERS}

The equations of motion for both Type A and Type B systems can be easily solved using a digital computer by employing the MIMIC Digital Simulation Program explained in Reference 7. This program was developed at Wright-Patterson Air Force Base to facilitate the application of digital computers to engineering problems. MIMIC is a program whose input language endows a digital computer, from the viewpoint of the user, with some significant advantages of an analog computer, in that the input relates directly to the physical nature of the problem, while virtually eliminating the time and amplitude scaling needed for analog computation.

The Type A system with the same exemplary parametric values previously used was also solved using the MIMIC Program and an IBM 7094 computer. The resulting answers were virtually 
identical and the plots of the MIMIC results are therefore the same as those shown in Figures 7 and 8. Figure 11 shows the input required by the MIMIC Program to solve the Type A system per Equations 11 and 12. This is the necessary input form with one punched card per line.

Figure 12 shows the input to the MIMIC Program for solution of a Type B system, Equations 17 and 18. The results of the Type $B$ system solution are shown in Figures 13 and 14. The input values were the same as in the previous examples with $l=4$ feet, and $M$ assumed infinite. A finite value of $M$ could be considered without excessive complication.

The MIMIC Program also lends itself to the solution of concurrent deployment of dissimilar appendages. The equations of motion would be more complicated and the computer input longer but the solution would still be much more rapid than by other methods. If some of the appendages are released sooner than others or locked in place while others are still deploying, then the problem could be solved in steps. This means the initial conditions and equations of motion would change at each abrupt change caused by appendages releasing or appendages locking in place. The MIMIC Program is a powerful and useful tool in the solution of the spinning spacecraft problem.
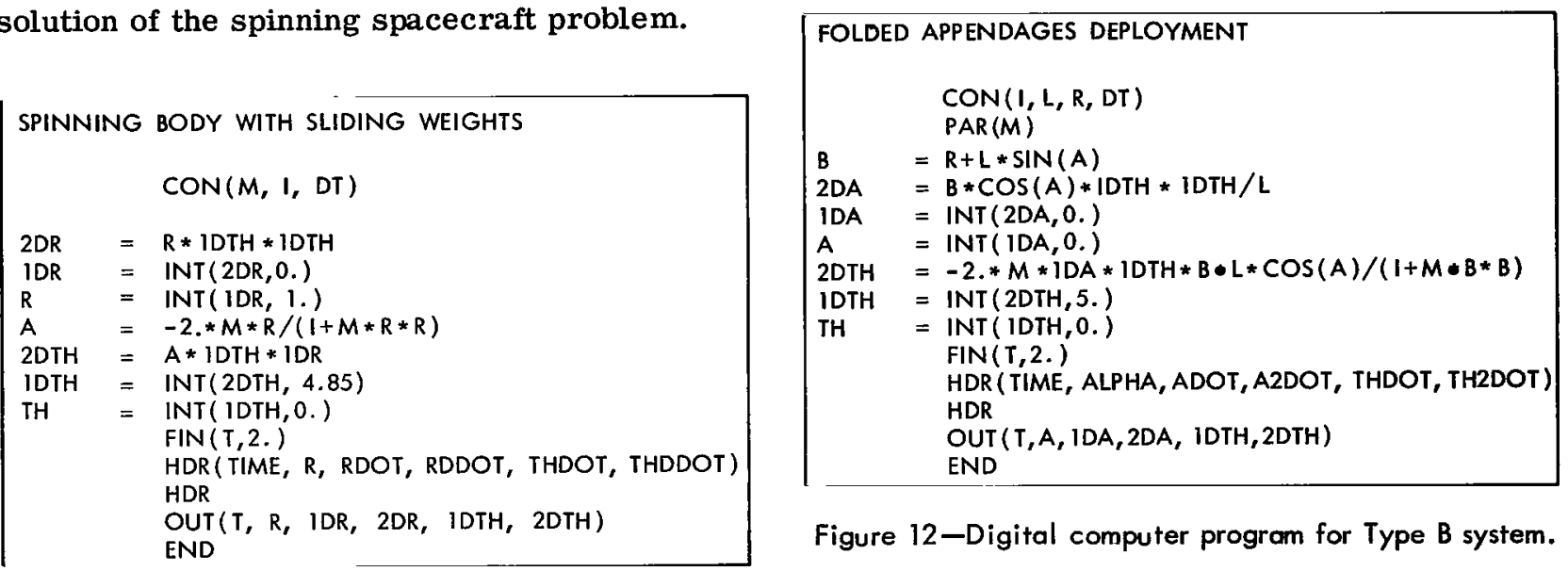

Figure 12-Digital computer program for Type B system.

Figure 11-Digital computer program for Type A system.

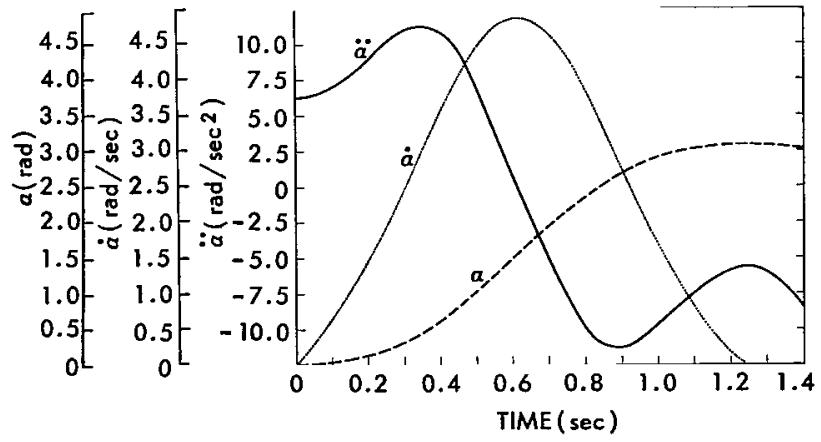

Figure 13-Digital computer solution for $a, \dot{\alpha}$, and $\bar{a}$ (Type B system).

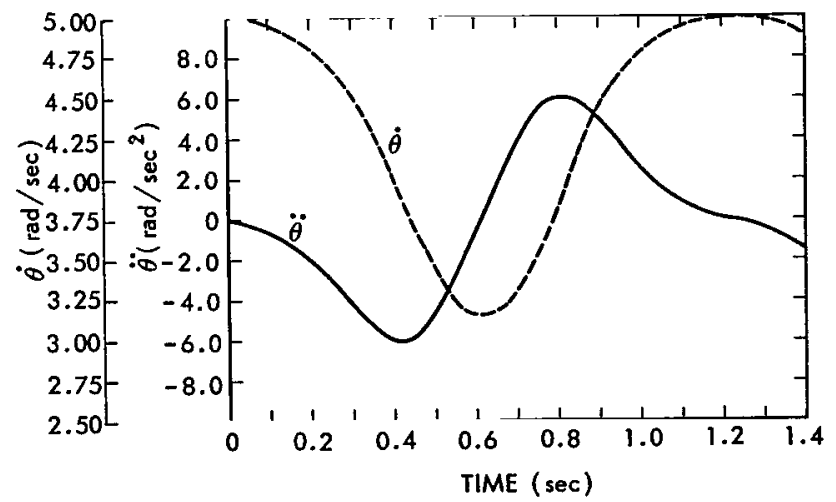

Figure 14-Digital computer solution for $\theta$ and $\ddot{\theta}$ (Type B system). 


\section{COMPARATIVE EVALUATION}

At this point it is pertinent to note that the same specific Type A problem was solved by three different and independent methods - the quasi-classical, with an analog computer and with a digital computer. All methods yielded essentially identical results. The actual system which led to the choice of these specific parameters also behaved approximately as predicted during test deployment (see test experience).

\section{EVALUATION OF ACTUAL SPACECRAFT SYSTEMS}

At this point it is pertinent to consider pragmatically the value of the preceding material. Real systems are less simple than those considered though deploying elements are often either essentially free to recede radially from the spin axis (Type A system) or essentially constrained to rotate out about fixed axes (Type B system). From discussion of these simple systems, the prospect of attempting a classical solution for a real system is discouraging especially as practical objectives often require only approximate answers to specific questions. Although even extremely complicated systems could be solved iteratively by sufficiently sophisticated analysis and computer facilities, the effort involved might well be inappropriate if acceptably accurate solutions leading to the same practical conclusions could be obtained relatively easily and quickly.

It is suggested that a sufficiently accurate and probably conservative (i.e., more severe than actual) estimate of dynamic behavior can be made by assuming that a real system has a functionally equivalent Type A or B system. Such an educated guess is certainly preferable to neglecting a possible problem area. It seems reasonable to postulate a rational equivalent model for many actual configurations.

\section{Examples}

1. Appendages which telescope radially outward. These are basically Type A systems and could be analyzed as such by assuming the mass of sliding elements concentrated at the mass center.

2. Single hinged appendages folded back against final stage booster, each as solar paddle arrays on many spacecraft. These are basically Type B systems with distributed deploying element mass. The total mass of each appendage could be considered concentrated at its mass center. Then the deployment could be analyzed as a Type B system.

3. Double hinged appendages such as shown in Figure 15. The validity of a simplified model for this kind of configuration is more dubious and controversial and largely depends on the relative mass and inertia of the inboard and outboard elements. In general, the inboard element is a Type B system while the outer element tends to behave like a Type A system, especially if its mass greatly exceeds that of the inner element. A Type A deployment is 
inherently more violent than a Type $B$ deployment and if a Type A system, with deploying mass equal to the total appendage mass, is assumed, conservative estimates of angular deceleration and deployment time will be obtained (i.e., Angular deceleration will be greater and deployment time less than for the actual system). The results would give some insight as to whether the transient dynamic effects during deployment might be significantly detrimental to the system. Before actual tests of a system as shown in Figure 15, behavior was pre-
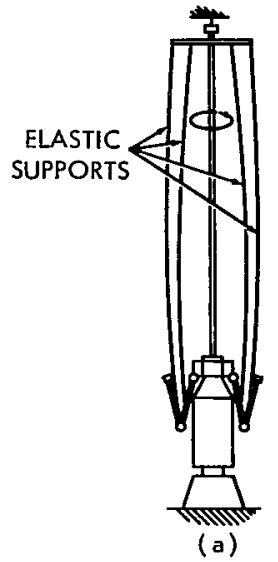

FOLDED

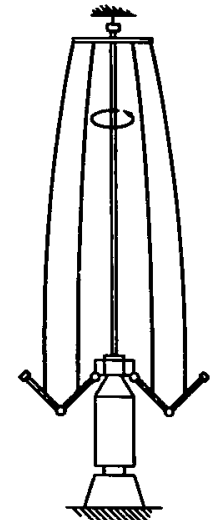

(b)

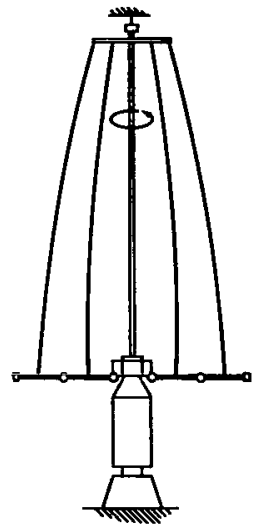

(c)

DURING DEPLOYMENT DEPLOYED

Figure 15-Gravity negation for double hinged boom deployment. dicted by assuming a Type $A$ system and the results obtained for duration of deployment and maximum angular deceleration were reasonably compatible with observed behavior during the tests.

4. Concurrent deployment of dissimilar appendages such as a set of inertia booms and a set of solar paddles. A system of this type requires consideration of the extent and nature of dissimilarity. For minor differences, a possible approach might be to assume that (1) the booms deploy from the initial spin rate with the paddles initially fully deployed and then (2) the paddles deploy from the initial spin rate with the booms initially fully deployed. For each case, develop time histories of accelerations, forces and torques, including the effects on the deployed paddles of boom deployment and vice versa. Then, assume (1) and (2) to be concurrent and add the two time histories for each parameter. The results would be conservative as the hypothetical situation involves greater energy and momentum transfer than the actual concurrent deployment.

5. Systems having springs to assist deployment and/or damping devices to retard deployment. A preliminary step would be to compare the energy dissipated in deployment (i.e., $E=(1 / 2)\left(I_{0} \dot{\theta}_{0}{ }^{2}\right)\left(1-I_{0} / I_{F}\right)$ with the energy supplied by the spring $(+e)$ or absorbed by the dampers (-e) during deployment. If "E" considerably exceeded "e", it would suffice to add "e" to "E" (for springs) or subtract "e" from "E" (for dampers) and calculate a new equivalent initial spin rate as $\left[\dot{\theta}_{0}^{2} \pm 2 \mathrm{e} / \mathrm{I}_{0}\left(1-\mathrm{I}_{0} / \mathrm{I}_{F}\right)\right]^{1 / 2}$. Then the system could be analyzed for the modified initial spin rate as if the springs and/or dampers were not present. Forsythe ${ }^{2}$ has applied similar logic to the problem of simulating zero gravity appendage deployment.

It should be emphasized that these examples are given only to illustrate the kind of logic that might be usefully applied with appropriate discretion. Any particular system should obviously be studied with full and complete knowledge and consideration of all germane factors. Also, this kind of "ballpark" analysis is more appropriate in conjunction with experimental testing of system 
behavior. If the system cannot be tested, a more rigorous analysis would be desirable though even then the methods discussed here might establish sufficient confidence in operational reliability to omit further study - or show that the design is fundamentally unreliable.

\section{Testing Experience}

Various spacecraft have been subjected to appendage deployment under conditions intended to adequately simulate orbital situations. The most intractable problem is probably the elimination of unrealistic external restraints, particularly those imposed by the ambient gravitational field. Four methods have been used to overcome this problem.

The first and certainly the best, when it can be used, is to have the deployment occur under "free fall" conditions. Unfortunately it is only feasible for short test durations for obvious reasons. The larger and more massive systems characteristically require longer times for deployment to occur and should not be subjected to the high accelerations which would necessarily be imposed by injection into, and recovery from, extended free fall trajectory.

The second method, of limited value but having the merit of simplicity, is based on determining the kinetic energy loss due to deployment which is $(1 / 2)\left(I_{0} \dot{\theta}_{0}{ }^{2}\right)\left(1-I_{0} / I_{F}\right)$ and assuming that this is absorbed by the structure at lockup into deployed configuration and appropriately divided between the various structural elements. Then each element is subjected to a shock pulse of appropriate energy content. This can often be done by setting up each appendage so that gravity causes its deployment with supplementary energy input from springs if necessary. The entire system is not subjected to spin, and the kinematics of deployment, including the possibly critically significant Coriolis effects are not simulated. However, such subsystem tests can reveal the ability of the structure to withstand "lock in" shock loads.

The third method, described by Forsythe (Reference 2) is to "overspin" the system to $\left(\theta_{0}+\delta\right)$ so that the excess available energy which is $(1 / 2) I_{0}\left(2 \delta \dot{\theta}_{0}+\delta^{2}\right)\left(1-I_{0} / I_{F}\right)$, equals, and therefore cancels, the gravity potential energy required to deploy the appendages. It is easy to apply and proper in some cases but for many cases it is entirely incorrect. For example, for a system shown in Figure 15 (with spin axis vertical) overspinning would cause excessively violent deployment of the outboard elements and possible failure to lock into fully deployed position. Also, for a basic Type A system, telescoping radially in a horizontal plane, no overspin would be indicated, but the weight of the deploying elements could well cause significant frictional resistance to deployment. Therefore this method should be used only with due consideration as to how well it actually duplicates orbital conditions. At best, it imposes equivalent "lock in" shock loads; the dynamics of deployment will always differ from those in orbit to some extent.

The fourth method is to actually provide a "counterforce" to each deploying element to cancel its weight. Elastic elements are commonly used, and they may either equate elastic energy with appropriate potential energy with a substantial force variation during deployment or, in more sophisticated applications, have a low enough spring rate (normally by virtue of length) that the force during deployment is relatively constant and essentially vertical, thereby approximating cancellation of gravity during deployment. 
Figure 15 illustrates a test arrangement used to achieve compensation of gravity to less than

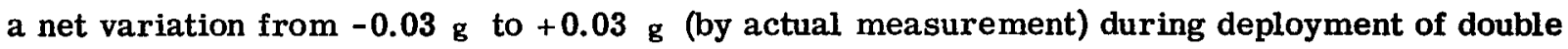
hinged appendages. The elastic elements used were cis-polyisoprene (natural gum rubber of high purity, as used in surgical tubing). This material was selected as having superior elastic properties and was used at elongations for minimum percentage deviation from tensions equal to the weights of the suspended elements. The radii to the upper attachment of the suspension elements were determined for minimum departure from verticality of their attachment to the appendage elements, after allowing for their spin-induced centrifugal deflection. The lower attachments were located at the mass center of each appendage element. Figure 15 shows a rigid extension of the spacecraft (to a height about $35 \mathrm{ft}$. above it) to drive the suspension beam - in a more recent application there was no physical connection except the elastic elements and the suspension beam remained oriented with the spacecraft within acceptable deviations.

It is believed that this test achieved a close approximation of zero gravity deployment. The total counterforce, as monitored by a load cell above the suspension beam, deviated only 6 percen during deployment. Unrealistic horizontal components of the suspension force did not, from high speed movie coverage, appear to exceed 7 percent of the tension in the element.

The test results tended to confirm predictions based on the foregoing analysis. A Type A system was assumed as the majority of the appendage mass was in the outer elements. The movies showed that the outer element mass center did, in fact, tend to recede radially in a horizontal plane from the spin axis. The duration of the deployment transient was approximately as predicted. The maximum angular deceleration from monitoring accelerometers installed tangentially on the central body was also approximately as predicted. The appendages (fiberglass booms) showed extreme deflections and hinge failure occurred during an overspeed (failure mode) test. The failure occurred during deployment and before latch in and was observably due to the Coriolis wrenching effect. Accelerometers were installed on the appendage elements to monitor transient accelerations; but the results were difficult to interpret because of the failure and because of the transient shocks induced by pyrotechnic boom release devices plus other high frequency indications of indeterminate origin.

To summarize the test results (including some not cited) tend to confirm the validity and utility of the preceding analysis. The accuracy of either analysis or testing cannot be precisely evaluated because the analysis applies to simplified and idealized systems and because the test systems, besides differing from the idealized models, were subject to imperfect environmental simulation. Since the analysis is based on incontrovertible basic principles, the computational errors would be minor, and the environmental simulation error was demonstrably minor, discrepancies between predictions and test results could be considered largely due to idealizing of the actual systems. Since these were no major discrepancies, the idealizing assumptions were apparently essentially valid.

\section{CONCLUSIONS}

The most significant conclusion is that the deployment dynamics of spinning spacecraft can be adequately simulated either analytically or by testing, but for maximum confidence the approaches 
should be complementary. Also, the solutions of simple systems, as described, can provide valuable insight into the dynamic behavior of more complex systems.

Both analysis or testing are subject to some degree of inaccuracy, the analysis being subject to simplifying assumptions and imprecise parametric data, and testing to imperfect simulation. Therefore either analysis or testing should demonstrate adequacy for conditions moderately more severe than those anticipated in flight. If this is done then the inaccuracies are tolerable, provided they are recognized.

Both analog and digital computers are valuable tools for analysis and the relative ease with which useful results may be deduced without recourse to computer facilities suggests that this approach should not be neglected even if computer facilities are available. It affords a useful concept of the nature of the dynamic phenomena involved.

\section{ACKNOWLEDGMENT}

The authors wish to thank Miss Rebecca Michaels for assistance in the analytical and computation phases of this study.

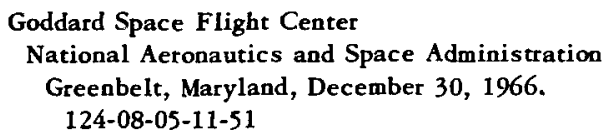

\section{REFERENCES}

1. Forsythe, R. W., "Analysis of Dissimilar Satellite Appendages During Erection", NASA Technical Memorandum X-938, January 1964.

2. Forsythe, R. W., "A Method for Simulating Zero Gravity Erection of Satellite Appendages", NASA Technical Memorandum X-937, January 1964.

3. Gale, E. H. and Gluck, R., "Final Report, Pioneer Spacecraft Appendage Deployment Verification Test", Thomas Ramo Woolridge Systems Report PP-IQ-02, April 1965.

4. Gluck, R. And Gale, E. H., "Motion of a Spinning Satellite During Deployment of Asymmetrical Appendages", Preprint 66-100, AIAA 3rd Aerospace Sciences Meeting, January 1966.

5. McCuskey, S. W., "An Introduction to Advanced Dynamics", Reading, Mass.; Addison-Wesley, 1959 , pp. 55-58.

6. "Handbook of Analog Computation", Electronic Associates, Inc., Publication No. 00-800-0001-1, October 1965.

7. Petersen, H. E. and Sansom, F. J., "MIMIC - A Digital Simulator Program", Directorate of Computation, Systems Engineering Group, Wright Patterson Air Force Base, Internal Memorandum 65-12, May 1965. 
Appendix A

\section{Influence of Central Body to Appendage Mass Ratio on Deployment Dynamics}

For tests involving components of motion of the deploying appendages parallel to the spin axis, there has been consideration of whether the support and restraint of the central body of the spacecraft, which effectively couples it to the comparatively infinite mass of the earth, compromises environmental simulation appreciably since in orbit (or in free fall tests) the central body mass is limited and the entire system is a free body. This general topic is discussed in this appendix and it is concluded that this factor can reasonably be ignored for the usual case where the central body mass in orbit is considerably greater than the total mass of deploying appendages.

Referring to the discussion of Type B systems, the orbital mass ratio is $\mathrm{M} / \mathrm{m}$. For Type A systems this ratio is immaterial, and, as previously mentioned, if $M=M / m=0$, a Type $B$ system behaves like a Type A system with faster deployment and higher values of tangential acceleration $a_{t}$. From Equation 26 the highest possible $a_{t}$ with $M / m=0$ exceeds the lowest possible $a_{t}$ with $\mathrm{M} / \mathrm{m}=\infty$ by a factor of $\left[\ell^{2} / \ell^{2}-\left(\mathrm{r}^{2}-\mathrm{r}_{0}\right)^{2}\right]^{1 / 2}$. For an arbitrarily chosen partially deployed position where $r-r_{0}=\ell / 2$ this factor would have a numerical value of 1.156. However, a similar comparison between $\mathrm{M} / \mathrm{m}=1$ and $\mathrm{M} / \mathrm{m}=\infty$ gives a numerical value of 1.069 and between $\mathrm{M} / \mathrm{m}=10$ and $\mathrm{M} / \mathrm{m}=\infty$ gives a numerical value of 1.011. The effect of mass ratio on deployment time is similarly minor for $\mathrm{M} / \mathrm{m}>1$ (from Equation 27). Deployment is slower for a greater value of $\mathrm{M} / \mathrm{m}$.

Therefore the support and restraint of the central body actually results in an undertest so far as the transient effects during deployment are concerned but to a minor degree for usual mass ratios (e.g., only 1.1 percent for $\mathrm{M} / \mathrm{m}=10$ ).

The other factor to be evaluated is the shock effect at lockup. From basic principles it is known that this effect has a constant energy content of $(1 / 2) I_{0} \theta_{0}^{2}\left(1-I_{0} / I_{F}\right)$, regardless of mass ratio, and since it is a collision between elements which terminate deployment (though energy is dissipated in compliance of the entire system) the question is essentially whether the "damage effect" of collision between two bodies depends only on the impact energy content or whether it is also influenced by the relative masses of the bodies. Their absolute and relative compliance, resilience and surface hardness are also pertinent factors but an adequate review of all these factors is a complex general problem beyond the scope of this paper. However it should be mentioned that precise orientation of appendages after deployment is often a design objective.

If a collision between two bodies has to dissipate a fixed amount of energy their relative velocity at impact is maximum when their masses are equal and minimum when one mass is infinite. The maximum relative velocity exceeds the minimum by a factor of $\sqrt{2}$. Between these 
two extremes, the relative velocity for a mass ratio of 10 is much closer to that for an infinite mass ratio than to that for a mass ratio of unity. However, the momentum change (i.e., impulse) to each body is greater for high mass ratio and lower relative velocity.

It follows that, for the appendage lock-in situation, an infinite (coupled to earth) mass ratio will produce a shock pulse of longer duration, but lower onset rate, than a true orbital mass ratio. The former sees more momentum change but at a slower rate. Both develop the same maximum forces and therefore the same stresses if elastic limits are not exceeded. These facts, though of academic interest, hardly warrant any definitive conclusion, except that the "test versus orbit" comparison of this factor for most systems would show it as probably insignificant as neither onset rate nor impulse would differ appreciably.

Therefore, restraint of the central body during test can usually be ignored for evaluation purposes. Also, the central body mass could usually be considered infinite for analytical evaluation. 
"The aeronautical and space activities of the United States shall be conducted so as to contribute ... to the expansion of buman knowledge of phenomena in the atmosphere and space. The Administration shall provide for the widest practicable and appropriate dissemination of information concerning its activities and the results thereof."

-Nationat aeronautics and Space Act of 1958

\section{NASA SCIENTIFIC AND TECHNICAL PUBLICATIONS}

TECHNICAL REPORTS: Scientific and technical information considered important, complete, and a lasting contribution to existing knowledge.

TECHNICAL NOTES: Information less broad in scope but nevertheless of importance as a contribution to existing knowledge.

TECHNICAL MEMORANDUMS: Information receiving limited distribution because of preliminary data, security classification, or other reasons.

CONTRACTOR REPORTS: Scientific and technical information generated under a NASA contract or grant and considered an important contribution to existing knowledge.

TECHNICAL TRANSLATIONS: Information published in a foreign language considered to metit NASA distribution in English.

SPECIAL PUBLICATIONS: Information derived from or of value to NASA activities. Publications include conference proceedings, monographs, data compilations, handbooks, sourcebooks, and special bibliographies.

TECHNOLOGY UTILIZATION PUBLICATIONS: Information on technology used by NASA that may be of particular interest in commercial and other non-aerospace applications. Publications include Tech Briefs, Technology Utilization Reports and Notes, and Technology Surveys.

Details on the availability of these publications may be obtained from:

SCIENTIFIC AND TECHNICAL INFORMATION DIVISION

NATIONAL AERONAUTICS AND SPACE ADMINISTRATION

Washington, D.C. 20546 